\title{
Role of the modern radiotherapy in the postoperative setting for esophageal cancer
}

\author{
Gian-Carlo Mattiucci, Francesco Cellini \\ Gemelli ART, Radiation Oncology Department, Università Cattolica del Sacro Cuore, Fondazione Policlinico A. Gemelli, Rome, Italy \\ Correspondence to: Francesco Cellini. L.go A.Gemelli 8, 00168 Rome, Italy. Email: francesco.cellini@policlinicogemelli.it. \\ Provenance: This is an invited Editorial commissioned by Section Editor Dr. Hongcheng Zhu, (Section Editor, Department of Radiation Oncology, \\ Fudan University Shanghai Cancer Center, Shanghai, China). \\ Comment on: Hwang JY, Chen HS, Hsu PK, et al. A propensity-matched analysis comparing survival after esophagectomy followed by adjuvant \\ chemoradiation to surgery alone for esophageal squamous cell carcinoma. Ann Surg 2016;264:100-6.
}

Submitted Sep 19, 2017. Accepted for publication Sep 28, 2017.

doi: $10.21037 /$ jtd.2017.10.07

View this article at: http://dx.doi.org/10.21037/jtd.2017.10.07

Hwang and colleagues recently reported on a propensity score matched (PSM) analysis to evaluate the role of adjuvant radiochemotherapy (RTCT) in esophageal cancer (1). They collected data from 1,095 esophageal squamous cell carcinoma (ESCC) patients. The investigated population both included patients who only underwent to surgery (group 1; 679 patients) and having also received adjuvant RTCT (group 2; 416 patients). The data about patients have been obtained from the Taiwan Cancer Registry database. Finally, 147 balanced patients per group were selected by PSM. The 3-year survival rate was $28.1 \%$ in group 1 and $44.9 \%$ in group 2, respectively. Interestingly, the multivariate analysis highlighted some factors resulted statistically significant predictors for clinical outcome: postoperative $\mathrm{T} 3$ and $\mathrm{T} 4$ (pT3/4) [hazard ratio (HR): 2.03 , 95\% CI: $1.38-2.97, \mathrm{P}<0.001$ ], postoperative positive lymph-nodes (pN+) (HR: 1.83, 95\% CI: 1.31-2.57, $\mathrm{P}=0.0004)$, tumor length over than $32 \mathrm{~mm}$ (HR: 1.93, 95\% CI: $1.33-2.79, \mathrm{P}<0.001)$, evidence of either microscopical or macroscopical residual (R1 or R2) at resection (HR: 1.75, 95\% CI: $1.15-2.66, \mathrm{P}=0.009)$, and administration of adjuvant RTCT (HR: 0.57, 95\% CI: 0.42-0.78, P<0.0001). Looking at the patient's characteristics, patients analyzed in group 2 were younger and presenting more advanced tumors compared with group 1 . Moreover in the group 2 there were more: $\mathrm{T} 3$ and $\mathrm{T} 4$ tumors $(77.6 \%$ vs. $28.6 \%)$, $\mathrm{N}+$ tumors $(76.8 \%$ vs. $20.3 \%)$, M1 tumors (6.3\% vs. $2.4 \%)$, stage III/IV tumors (68.5\% vs. $15.9 \%)$, large-sized tumors (45.6 vs. $30.9 \mathrm{~mm}$ ) and poorly differentiated tumors
(32.6\% vs. $23.4 \%$ ). The rate of residual tumor at resection margin (R1/2 resection) was also higher in the group 2 (18.6\% vs. $6.9 \%$ ). Authors' report that around $45 \%$ of the presentations were preoperatively sited in lower esophagus in both groups, but do not specifically account for the junctional presentations. We could summarize that such evidence suggests how postoperative RTCT seems able to compensate for adverse features individuated after surgery, including pT3/4, pN+, more extended tumor length, R1/2, more advanced histological grade. First of all, looking at the presented paper, the reproducibility of the results for all the patients could be questioned since derived from an Eastern population. The issue must be taken in consideration but the presented results are also in line with a previously published experience by Rice and colleagues, highlighting the advantage by administration of adjuvant RTCT over surgery alone in a smaller single-center Western population experiment performed with a similar methodology of propensity-matched comparison for case selection (2). That study involved 31 and 52 patients undergone to postoperative RTCT and surgery alone, respectively; both adenocarcinomas (approximately $84 \%$ in both arms) and squamous lesions (approximately $16 \%$ in both arms) were included; pT1-4 pN0-1 M0-1 lesions were collected. The trial published by Rice et al. strictly aimed to define the clinical efficacy of adjuvant RTCT on the global dataset: no focus on patient's characteristics was driven. Conversely, in the report of Hwang et al. a subgroup analysis focused the advantage derived by receiving postoperative RTCT 
in specific subset of patients: that is significantly gained for each subgroup including $\mathrm{R} 1 / 2$ resections, T3/4, N+, longer than $32 \mathrm{~mm}$, poorly differentiated lesions. Nowadays, on the basis of some clear literature evidences (3-5) there is a quite wide consensus for the treatment option of esophageal lesions, including for the locally advanced squamous ones: it should be nevertheless noted as the rate of squamous presentations in the CROSS trial is approximately $23 \%$. Thus, the data presented by Hwang and coworkers could allow a reflection whether could there be room for a modification of the indications provided by the currently available guidelines. The last release version of the National Comprehensive Cancer Network (NCCN) guidelines suggests preoperative RTCT as primary treatment option for non-cervical locally advanced respectable presentation (i.e., cT1b-T4 anyN) of ESCC (6). That implies that all the patients should be theoretically referred to preoperative RTCT in the clinical practice. Moreover RTCT is the recommended option when dealing with the case a non-preoperatively irradiated patients if reporting a microscopically or macroscopically non-radical surgery (i.e., R1-2). On the contrary if a patient already received RTCT before surgery, the proposed options include observation only, palliative treatment or best supportive cares. It should be highlighted that the results presented by Hwang and coworkers are based on patient data obtained from Taiwan Cancer Registry database, which is a national populationbased database: although interesting and based on a good methodology and big number of collected patients it has not the evidence level of a randomized trial. Thus, three main types of considerations could be addressed regarding the challenges that such evidence arises: may we potentially reconsider the general indication of upfront preference for preoperative RTCT? Should we offer postoperative RTCT to a wider population of patients? Is there room for further integration of postoperative RTCT as an intensification of multimodal treatment?

First issue: should we reconsider the indication for preoperative RTCT over the postoperative one? Preoperative RTCT enables early treatment of distant metastases while simultaneously treating the primary disease, facilitates definition of radiotherapy target volumes, and may allow resection of advanced disease. It has, however, some toxicity and, although data about the effect of such toxicity on the clinical global outcome are still unclear (7), it may reduce the ability of some patients to tolerate resection. Postoperative RTCT allows for early surgical debunking, rapidly addresses dysphagia, and allows for RTCT based on accurate pathologic staging, but delays systemic treatment. This is certainly a hard issue since indirect comparison of evidence in literature is not always applicable: often the series administering adjuvant RTCT tend to reserve that to more advanced presentations (also including the presence of surgical residual). Few available reports recently investigated that issue in literature. Looking at them in detail, two have some issues about the characteristics of the involved patients: one only including T4 lesions, administering low radiotherapy dose and not specifying the histology (squamous or adenocarcinoma) (8); or both enrolling the two histology like in the Surveillance, Epidemiology, and End Results (SEER) cancer registry base paper authored by Hong et al. (9). Nevertheless these 2 evidences support the role of preoperative RTCT over the postoperative one. Similarly to the paper we are discussing (1), other two reports are national populationbased from the Taiwan Cancer Registry: Chen et al. collected 234 patients with ESCC, divided into three groups (preoperative RTCT, postoperative RTCT and Surgery alone) (10). They found that bot the RTCT-administering arm were significantly superior to the surgery-only option. In contrast, the survival was similar between the preoperative RTCT and postoperative RTCT groups $(\mathrm{P}=0.544)$. Moreover, patients with clinical T3/4 stage tumors and those with a tumor size greater than $5 \mathrm{~cm}$ were more likely to demonstrate an overall survival benefit from preoperative RTCT compared with postoperative RTCT, suggesting a more founded role for such an approach for these clinical presentations. Hsu and colleagues applied the PSM method to 572 patients with ESCC whose data were collected by the Taiwan Cancer Registry, balancing the analyzed population among who underwent to either preoperative or postoperative RTCT, enrolling T1-4, $\mathrm{N} 0 /+, \mathrm{R} 0 / 1 / 2$ presentations (11). This analysis did not find a significant difference favoring one approach over the other: interestingly, there was a non-significant trend for preoperative administration of RTCT for all the analyzed clinical outcomes (i.e., overall and disease-free survival).

A randomized trial facing approximately 80 patients in each of its 3 arms (preoperative RTCT, postoperative RTCT, surgery alone, respectively) was provided by Lv et al. (12). In their experience local recurrence rate significantly favored the preoperative RTCT over the others options (preoperative $11.3 \%$, postoperative $14.1 \%$ and surgery alone $35 \%$, respectively; $\mathrm{P}<0.05$ ). On the contrary, similarly to the previously mentioned papers, the clinical outcome (specifically the 1-, 3-, 5-, 10-year overall and 
disease-free survivals) did not differ significantly, although the preoperative RTCT always had a non-significant favored trend. We can summarize that though there is some conflicting results, there is still not probably enough data (particularly not strong enough) to support the equivalence of postoperative to preoperative RTCT; as most of the authors of the mentioned studies conclude: further evidence are still needed to investigated such a relevant point.

Second issue: should we offer postoperative RTCT to a wider population of patients? We mentioned that the NCCN guidelines currently only advice postoperative RTCT for patients with evidence of residual disease after surgery (R1/2). The paper from Hwang et al. actually questions that point since in their experience also pT3/4, $\mathrm{pN}+$, larger sized lesion and poorly differentiated tumors were more likely to demonstrate survival benefit from adjuvant RTCT. Similarly, other published experiences highlighted that some factors other than resection margin status could be associated to an advantage by receiving postoperative RTCT, in particular for the positive nodal status $(13,14)$, specifically in the field of $\operatorname{ESCC}(15,16)$ and when presenting extracapsularity (17). It is not clear if such characteristics could be incorporated into new guidelines: if the presence of a certain level of post-surgical residual is clearly associated with a worse clinical outcome, if an adjuvant therapy should be routinely incorporated into the preferable clinical practice in presence of $\mathrm{pN}+$ and/or larger lesions irrespectively if a $\mathrm{R} 0$ resection was achieved, need probably a more robust analysis. Finally a third issue can be briefly mentioned: the chance for a further integration of postoperative RTCT to intensification the standard multimodal treatment. A recent report from Hsu et al. retrospectively analyzed (by PSM) a group of 64 patients with ESCC cancer who showed poor pathological response to preoperative RTCT: all the investigated population obtained a R0 resection, but showed persistent nodal disease or a postoperative ypT stage greater than or equal to the pretreatment clinical T stage (18). Authors meant to investigate whether postoperative adjuvant therapy could provide a clinical impact by providing to the two groups of patients either surveillance or adjuvant therapy (mostly represented by a second course of RTCT delivering 23.4 to $30 \mathrm{~Gy}$ - up to a total dose of 60 to $65 \mathrm{~Gy}$ ). Compared with those undergoing surveillance, the 3 -year disease free survival was significantly improved ( $45 \%$ vs. $22.3 \%$; $\mathrm{P}=0.022$ ), but authors warned about the need for a carefully weighed against a potential increase in the risk of treatmentrelated death.
Also this last treatment opportunity seems intriguing but strictly needs to be better exploited by a bigger and more robust prospective evaluation before to be recommended for the common clinical practice. Unfortunately it is not always possible to plan and perform a randomized trial with an adequate strength: collecting enough patients can be difficult, and the time needed to plan, set and complete a trial can be conflicting with the urgency of some answers; moreover the rapidity of the scientific evolution could challenge certain schedules or chosen drugs. The rapid and continuous accumulation of new evidences on such an amount of different sources (imaging, genetic, clinical, trials) determines the urgency for solutions able both to quickly interpret the evidences derived from new data and to confirm on independent and wide confirmatory datasets the upcoming results of new hypotheses derived from research (19). The use of large and shared databases for data-mining added to implementation of new technologies applied to data interpretation could be a valid option (20). Being able in the close future to rapidly handle big-data from different sources and to address a research featured by algorithms based on learning machines could enhance our chances to investigate and validate predictive models able to answer highly complex questions. By now, remaining strong the evidence privileging the choice for preoperative RTCT in the routine clinical practice, the experience of Hwang and colleagues suggest us to further study the issue of RTCT, particularly in the perspective of patient selection, timing with surgery and integration with systemic agents, possibly in the setting of randomized controlled clinical trials.

\section{Acknowledgements}

None.

\section{Footnote}

Conflicts of Interest: The authors have no conflicts of interest to declare.

\section{References}

1. Hwang JY, Chen HS, Hsu PK, et al. A propensitymatched analysis comparing survival after esophagectomy Followed by adjuvant chemoradiation to surgery alone for esophageal squamous cell carcinoma. Ann Surg 2016;264:100-6.

2. Rice TW, Adelstein DJ, Chidel MA, et al. Benefit 
of postoperative adjuvant chemoradiotherapy in locoregionally advanced esophageal carcinoma. J Thorac Cardiovasc Surg 2003;126:1590-6.

3. van Hagen P, Hulshof MC, van Lanschot JJ, et al. Preoperative chemoradiotherapy for esophageal or junctional cancer. N Engl J Med 2012;366:2074-84.

4. Oppedijk V, van der Gaast A, van Lanschot JJ, et al. Patterns of recurrence after surgery alone versus preoperative chemoradiotherapy and surgery in the CROSS trials. J Clin Oncol 2014;32:385-91.

5. Shapiro J, van Lanschot JJB, Hulshof MCCM, et al. Neoadjuvant chemoradiotherapy plus surgery versus surgery alone for oesophageal or junctional cancer (CROSS): long-term results of a randomised controlled trial. Lancet Oncol 2015;16:1090-8.

6. Ajani JA, D'Amico TA, Almhanna K, et al. Esophageal and esophagogastric junction cancers. Version 1.2015.J Natl Compr Canc Netw 2015;13:194-227.

7. Kumagai K, Rouvelas I, Tsai JA, et al. Meta-analysis of postoperative morbidity and perioperative mortality in patients receiving neoadjuvant chemotherapy or chemoradiotherapy for resectable oesophageal and gastrooesophageal junctional cancers.Br J Surg 2014;101:321-38.

8. Fujita H, Sueyoshi S, Tanaka T, et al. Prospective nonrandomized trial comparing esophagectomy-followed-bychemoradiotherapy versus chemoradiotherapy-followedby-esophagectomy for T4 esophageal cancers. J Surg Oncol 2005;90:209-19.

9. Hong JC, Murphy JD, Wang SJ, et al. Chemoradiotherapy before and after surgery for locally advanced esophageal cancer: a SEER-Medicare analysis. Ann Surg Oncol 2013;20:3999-4007.

10. Chen HS, Wu SC, Hsu PK, et al. The prognostic impact of preoperative and postoperative chemoradiation in clinical stage II and III esophageal squamous cell carcinomas: a population based study in Taiwan. Medicine (Baltimore) 2015;94:e1002.

11. Hsu PK, Chen HS, Liu CC, et al. Pre- versus

Cite this article as: Mattiucci GC, Cellini F. Role of the modern radiotherapy in the postoperative setting for esophageal cancer. J Thorac Dis 2017;9(11):4212-4215. doi: 10.21037/ jtd.2017.10.07 postoperative chemoradiotherapy for locally advanced esophageal squamous cell carcinoma. J Thorac Cardiovasc Surg 2017;154:732-740.e2.

12. Lv J, Cao XF, Zhu B, et al. Long-term efficacy of perioperative chemoradiotherapy on esophageal squamous cell carcinoma. World J Gastroenterol 2010;16:1649-54.

13. Xu Y, Liu J, Du X, et al. Prognostic impact of postoperative radiation in patients undergoing radical esophagectomy for pathologic lymph node positive esophageal cancer. Radiat Oncol 2013;8:116.

14. Shridhar R, Weber J, Hoffe SE, et al. Adjuvant radiation therapy and lymphadenectomy in esophageal cancer: a SEER database analysis. J Gastrointest Surg 2013;17:1339-45.

15. Chen J, Pan J, Liu J, et al. Postoperative radiation therapy with or without concurrent chemotherapy for nodepositive thoracic esophageal squamous cell carcinoma. Int J Radiat Oncol Biol Phys 2013;86:671-7.

16. Hsu PK, Huang CS, Wang BY, et al. Survival benefits of postoperative chemoradiation for lymph node-positive esophageal squamous cell carcinoma. Ann Thorac Surg 2014;97:1734-41.

17. Wang ZW, Luan ZP, Zhang W, et al. Postoperative chemoradiotherapy improves survival in esophageal squamous cell cancer with extracapsular lymph node extension. Neoplasma 2014;61:732-8.

18. Hsu HY, Chao YK, Hsieh CH, et al. Postoperative adjuvant therapy improves survival in pathologic nonresponders after neoadjuvant chemoradiation for esophageal squamous cell carcinoma: a propensitymatched analysis. Ann Thorac Surg 2016;102:1687-93.

19. Valentini V, Cellini F. New perspectives in treatment decision for integrated management of rectal cancer: multimodal research for multimodal treatments. G Chir 2014;35:113-6.

20. Roelofs E, Dekker A, Meldolesi E, et al. International data-sharing for radiotherapy research: an open-source based infrastructure for multicentric clinical data mining. Radiother Oncol 2014;110:370-4. 